\title{
More Basic Confocal Microscopy: A Tutorial
}

\author{
Robert L. Price* and W. Gray (Jay) Jerome**
}

* Department of Developmental Biology and Anatomy, School of Medicine, University of South Carolina, Columbia, SC 29208

**Department of Pathology, Vanderbilt University Medical Center, Nashville, TN 37232

This is a follow-up tutorial to the successful confocal tutorial presented last year at M\&M 2002. Confocal microscopy has become a primary method in many laboratories for visualizing microscopic structure in the third and fourth dimensions. However, in many cases individual users are expected to operate complex confocal scanning laser microscopes (CSLM) without being given the opportunity to learn basic principals. These basics include optimizing specimen preparation, basic fluorescent microscopy, the functions of the various components of a confocal system, methods for optimizing digital image capture and differences in digital image formats. All too often naive users are given only the rudiments of CSLM operation and left on their own to capture images. When conditions are not optimized rationally the result can be spurious data and images that are less than publication quality.

The material in this tutorial will be aimed at the advanced beginner and intermediate level users of confocal microscopes. The tutorial will address many of the concerns a researcher must be aware of in properly preparing a specimen for confocal imaging and setting up the user adjustable parameters of a CSLM to optimize image collection. Topics to be covered include: A) a comparison of wide-field and confocal microscopy, B) advantages and disadvantages of using and maintaining CSLM systems, C) specimen preparation and characteristics of fluorescent dyes, D) components of a generic confocal system, E) considerations in collecting a quality digital image with a CSLM, F) imaging formats and G) available electronic and print resources.

While each of us is familiar with individual makes of instruments an effort will be made to present basic generic material that can be applied to all makes and models of confocal systems. Specimen preparation may be the most important aspect of collecting meaningful results from a confocal microscopy experiment. With a poorly prepared specimen it will be impossible to collect a good image. However, with a well prepared specimen one can always go back and collect images several times so that each image or series of images is of good quality and appropriate for publication. Examples of topics to be considered during the discussion on specimen preparation include a variety of fixation protocols and the advantages and disadvantages of typical aldehyde fixation in comparison to other methods of fixation, approaches to blocking specimen autofluorescence, and how to select appropriate fluorochromes when performing double and triple label protocols.

While specimen preparation may be the most important aspect of obtaining quality confocal images, a thorough understanding of the role of the major components in the system is essential in setting up an instrument to collect quality images. During the tutorial we will discuss the various lasers, dichroic filters, mirrors, and PMTs that are available and the critical role each plays in obtaining good images. We will also review software settings for each of these components with regard to optimizing data quality. Included in the discussion will be the use of test specimens to set up the operating parameters of the confocal properly. These are critical to 
obtain digital images with a full range of pixel values for optimal presentation of image detail. An example of the type of images that will be presented is shown in Figure 1.

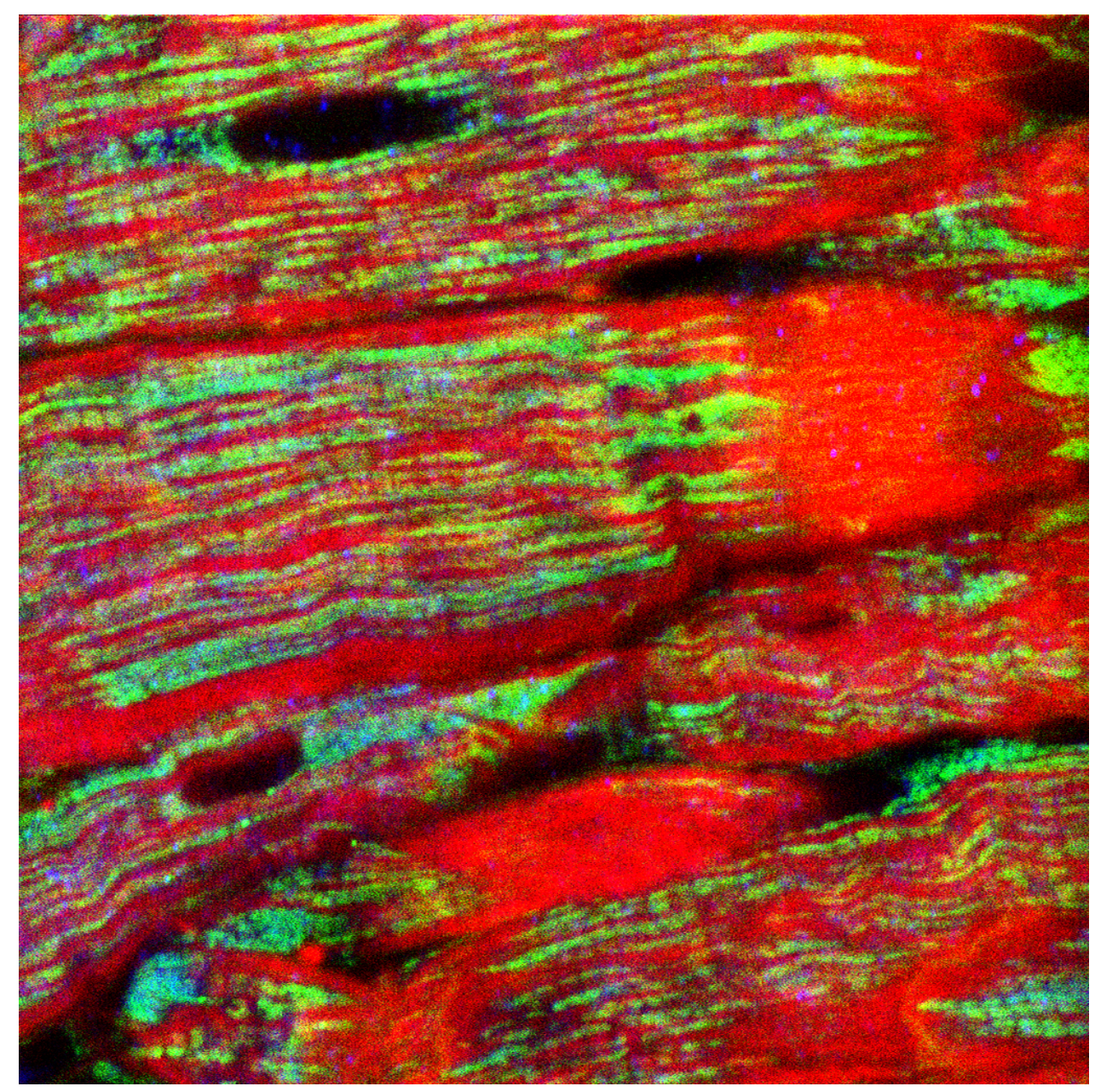

Figure 1. Vibratome section of mouse heart triple stained with rhodamine phalloidin (red, mitotracker green and cytochrome $\mathrm{C}$ (blue) to show colocalization of mitochondria and cytochrome C. 